\title{
Strength and power in children with low motor performance scores: a descriptive analysis
}

\author{
CASSIO V. RUAS 1 | CODY PUNT² | RONEI S. PINTO'1 | MARCIO A. OLIVEIRA²
}

${ }_{1}^{1}$ Physical Education School, Federal University of Rio Grande do Sul, Porto Alegre, Brazil | 2 School of Public Health, University of Maryland, MD, EUA.

Correspondence to: Cassio V. Ruas, Physical Education School, Federal University of Rio Grande do Sul, UFRGS, Felizardo Street, 750 - Jardim Botânico Porto Alegre 90690-200 Porto Alegre/RS, Brazil

e-mail: cassio ruas@hotmail.com

\begin{abstract}
AT A GLANCE
Neuromechanical underlying mechanisms may be related with motor coordination and control difficulties experienced by children with LMP. The current study tested knee isometric and isokinetic strength, handgrip strength, and explosive jump power. The results demonstrated lower descriptive mean scores of children with LMP as compared to the TD peers. In addition, the findings indicated delay in the process of developing strength and power
\end{abstract}

\section{ABBREVIATIONS}

$D C D$ - developmental coordination disorder

LMP - low motor performance

TD - typically developing children

PT - peak torque

\begin{abstract}
Children with low motor performance scores (LMP), such as those who have Developmental Coordination Disorder (DCD), demonstrate inability in performing motor tasks at the same level as typically developing (TD) average age peers. Information processing problems and perceptual deficits have been reported as the main causes for this discrepancy, but not much attention has been given to the neuromechanical underlying mechanisms related to motor difficulties. This study aimed to explore descriptive average values of strength and explosive power tasks in children with LMP scores, and individually analyze their strength and power outputs with age progression. Twenty-four children were tested on the Movement Assessment Battery for Children (MABC-2). Five children scored below the $15^{\text {th }}$ percentile (10.8 $\pm 1.7 \mathrm{yrs})$ and were placed in the LMP group $(n=5)$. Nineteen children $(10.7 \pm 1.7 \mathrm{yrs})$ were included on the TD group $(n=19)$. Participants' isometric, isokinetic and handgrip strength and explosive power were tested. The group of children with LMP presented inferior descriptive mean scores as compared to the group with TD children at knee isometric and isokinetic, handgrip, long jump and vertical jump tests. In addition, the results demonstrated that strength and power increase with age for those children with LMP. The findings suggest that children with LMP may be delayed in developing strength and power, which may be affecting their ability to perform gross and fine motor skills at the same level as their TD peers. We recommend that intervention programs for children with LMP include specific motor tasks and exercises focusing on neuromuscular coordination as well as ability to manage muscle strength and power levels.
\end{abstract}

KEYWORDS: Developmental coordination disorder | low motor performance | children | strength | power | force

\section{INTRODUCTION}

Difficulties in motor control and coordination affect 5-6\% of school-aged children ${ }^{1,2}$ and are identified by low motor performance scores (LMP). These difficulties are typically classified in the literature as Developmental Coordination Disorder (DCD), but can also be related with other developmental disorders, such as autism and attention deficit hyperactivity disorder (ADHD) ${ }^{3}$. The hypotheses regarding the causes of motor coordination and control problems, such as $D C D$, state that motor learning skills may affect automatization deficits or errors in creating accurate movements according to predictive movements, both as consequences of cerebellum pathology ${ }^{1}$. However, this is not a consensus. Motor task execution and proprioception problems have also been theorized as possible causes of such motor difficulties ${ }^{4}$.

Researchers have also been questioned if the lack of physical capacity is one of the main causes of poor motor functioning in children with this LMP2. Children with $D C D$, for example, often encounter problems performing normal daily activities, such as brushing teeth or tying shoes ${ }^{1}$. Additionally, DCD has been related to obesity, coronary vascular disease, 
and lack of physical fitness, balance and strength ${ }^{1,4-7}$ impacting other aspects of their development such as social and emotional factors ${ }^{8,9}$. From a developmental perspective, it has been found that although some motor difficulties in children may reduce as age progresses, there is a great possibility they may continue into adolescence and adulthood ${ }^{1,5}$. Although strength and power exercises have been suggested to be included in intervention programs for children with $L M P^{5,10,11}$, the European Academy for Childhood Disability (EACD) $)^{12}$ has not given sufficient attention to these variables when proposing therapeutic improvement activities for children with DCD.

Despite of the extensive literature reporting potential causes of lower levels of motor skill performance in children ${ }^{5,6,8,13,14}$, the hypothesis of potential neuromuscular deficits has not been fully explored ${ }^{5,8}$. Only a few studies have shown that difficulties with motor coordination and control could be due to the struggles related to strength, power and force production and regulation, 5,815-18. For example, the fine maximal and submaximal motor strength (e.g. maximum index finger and thumb torque production) has been investigated in children with DCD, who demonstrated to have the same level as typically developing peers, although the torque control during these tasks was weak and variable ${ }^{8,15}$. Children with DCD have also shown to have decreased lower limb gross motor maximal strength ${ }^{5}$ and explosive power ${ }^{9,13}$. In addition, two studies ${ }^{10,17}$ have found motor coordination improvements with strength training in children with LMP. These findings provide a foundation to hypothesize that the lack of neuromuscular strength power and control may play a critical role in motor coordination deficiencies. This could switch the cause and effect issues related to difficulties in motor control and coordination. Therefore, the aim of this study was to explore descriptive mean values of strength and explosive power test scores in children with LMP, and individually analyze their power and strength outputs by age progression in relation to typically developing children.

\section{METHODS}

Participants

Twenty-four male participants were included in this study. Potential participants were screened in a two-step process: a) children were reported by their teachers as experiencing difficulties in school motor activities, and b) assessment by the Movement Assessment Battery for Children (MABC-2). Five children scored below the $15^{\text {th }}$ percentile $(10.8 \pm 1.7 \mathrm{yrs})$ and were placed in the LMP group $(n=5)$. Nineteen children $(10.7 \pm 1.7 \mathrm{yrs})$ were included on the TD group $(n=19)$. One child $(11 \mathrm{yrs})$, included on the LMP group, scored below the $5^{\text {th }}$ percentile. All participants who scored above the $30^{\text {th }}$ percentile were placed in the TD group. Parents or guardians of all participants signed consent forms. The Institutional Review Board at the University of Maryland approved the protocol.

\section{Procedures}

Body mass was measured prior to testing. Subjects' lower and upper limb strength were tested in isometric, isokinetic and handgrip tasks. Isometric and isokinetic right knee strength were measured by a computer-controlled $\mathrm{KinCom} \otimes$ dynamometer. Participants were comfortably strapped into the chair with their right leg fastened to the lever arm. The left limb was not tested since there are no significant side-to-side differences in knee muscle peak torques for children ${ }^{16,19}$. They completed one set of five-second isometric right knee extension contractions at $90^{\circ}$ (knee totally flexed), and two sets of three repetitions of isokinetic knee extension and flexion tasks in a $0^{\circ}-60^{\circ}$ range, at two different speeds $\left(60^{\circ} \cdot \mathrm{s}^{-1}\right.$ and $\left.120^{\circ} \cdot \mathrm{s}^{-1}\right)$. The repetitions and angles were randomly selected and subjects practiced a warm-up attempt at each angle and speed prior testing. Between each set the participants were given 60 seconds rest intervals. The subjects' maximum handgrip strength for each hand was recorded by a handgrip test on a T.K.K. 5001 Grip-A handgrip dynamometer. The greatest score within three trials was recorded for each hand and summed for a final handgrip score.

Subjects were also tested at explosive power tasks, such as vertical jump and standing long jump tests. To measure the vertical jump, a tape measurer was set vertically along a flat wall. The subjects were asked to stand perpendicularly next to the wall and reach their dominant hand as high as possible along the tape measure. This reach height value was then recorded. Participants were instructed to complete three maximum vertical jump attempts and reach the dominant hand as high as possible, hitting the wall at the apex of the jump. The highest height of their hand along the wall at the apex of the jump within the attempts was recorded and subtracted by the reach height for the final score. For the long jump test, a tape measure was placed along the floor. Participants were instructed to begin with their heels at the zero point, and jump, with both feet, as far as possible. The measure recorded was the distance traveled by the heel of the foot that traveled the least distance. Three maximal attempts were completed and the highest score was recorded. Instructions and familiarization trials were given before all testing trials. Subjects had 3 minutes between trials and 5-minute rest intervals between each explosive power tests.

Data Analysis

The strength tests were normalized by subjects' body weight (absolute mass) ${ }^{5}$. To compare isokinetic and isometric strength values, descriptive mean and standard deviation values were calculated for both the TD and LMP groups. The 
greatest attempt of each explosive power test was also recorded. All descriptive analyses were performed with SPSS v.20 (Statistical Package for Social Sciences, Chicago, IL, USA). However, no statistical comparisons for trends and differences were made.

\section{RESULTS}

Age and body mass average measures for each group are presented on Table 1 . The LMP and TD isometric peak torque (PT) knee extension mean values at $90^{\circ}$, and the isokinetic PT knee flexion and extension mean values at $60^{\circ} \cdot \mathrm{s}^{-1}$ and $120^{\circ} \cdot \mathrm{s}^{-1}$ are presented in Table 2. The greatest scores for handgrip, and explosive power vertical jump and standing long jump tests are presented in Table 3. The individual LMP scores and TD group mean scores of each test are showed in Figures 2 and 3 , and are presented along the $x$-axis by age.

All tests revealed descriptive higher mean values in the TD group compared to LMP group (Tables 2 and 3). In addition, all tests demonstrate a clear trend of increases in strength of children with LMP with the progression of age, and do not apparently differ from the TD mean progression with age (Figures 1 and 2), except for knee flexion at $60^{\circ} \cdot \mathrm{s}^{-1}$ (c) (Figure 1), in which the group with LMP presents lower scores than the mean TD. Children with LMP present the greatest variability in the vertical jump (g) and long jump ( $h$ ) tests (Figure 2). Furthermore, subject number 3 with LMP, who is around one year and a half older than the mean age of children with TD, demonstrated his lowest scores compared to mean TD at knee isokinetic flexion at $60^{\circ} \cdot \mathrm{s}^{-1}$ (c) (Figure 1), and vertical jump (g) and long jump (h) tests (Figure 2). Subject number 2 with LMP, who is around one year younger than the mean age of the TD children, demonstrated to have greater scores than the mean TD at knee isokinetic extension at $120^{\circ} \cdot \mathrm{s}^{-1}(\mathrm{~d})$ (Figure 1).

Table 1: Means ( \pm SD) of age and body mass for children with low motor performance scores (LMP) and traditionally developing (TD) children.

\begin{tabular}{ccc} 
& Age $(\mathrm{yrs})$ & Body Mass $(\mathrm{kg})$ \\
\hline TD & $10.7 \pm 1.6$ & $38.8 \pm 11.3$ \\
\hline LMP & $10.9 \pm 1.3$ & $62.3 \pm 13.8$
\end{tabular}

Table 2: Means ( \pm SD) of knee extension isometric peak torque (PT) at $90^{\circ}$, and knee flexion and extension isokinetic PT at $60^{\circ} \cdot \mathrm{s}^{-1}$ and $120^{\circ} \cdot \mathrm{s}^{-1}$ for children with low motor performance scores (LMP) and typically developing children (TD).

\begin{tabular}{|c|c|c|c|c|c|}
\hline & Isometric & \multicolumn{2}{|c|}{ Isokinetic } & \multicolumn{2}{|c|}{ Isokinetic } \\
\hline & $\begin{array}{l}\text { PT Extension } \\
(\mathrm{N} \cdot \mathrm{m} / \mathrm{kg})\end{array}$ & $\begin{array}{l}\text { PT Extension } \\
(\mathrm{N} \cdot \mathrm{m} / \mathrm{kg})\end{array}$ & $\begin{array}{c}\text { PT Flexion } \\
(\mathrm{N} \cdot \mathrm{m} / \mathrm{kg})\end{array}$ & $\begin{array}{l}\text { PT Extension } \\
(\mathrm{N} \cdot \mathrm{m} / \mathrm{kg})\end{array}$ & $\begin{array}{c}\text { PT Flexion } \\
(\mathrm{N} \cdot \mathrm{m} / \mathrm{kg})\end{array}$ \\
\hline & $90^{\circ}$ & \multicolumn{2}{|c|}{$60^{\circ} \cdot s-1$} & \multicolumn{2}{|c|}{$120^{\circ} \cdot s-1$} \\
\hline TD & $8.7 \pm 1.8$ & $0.94 \pm 0.28$ & $0.96 \pm 0.29$ & $0.65 \pm 0.21$ & $0.73 \pm 0.91$ \\
\hline LMP & $6.2 \pm 2.2$ & $0.89 \pm 0.30$ & $0.92 \pm 0.30$ & $0.54 \pm 0.13$ & $0.61 \pm 0.28$ \\
\hline
\end{tabular}

Table 3: Means ( \pm SD) of vertical jump, long jump, and handgrip strength tests for children with low motor performance scores (LMP) and typically developing children (TD).

\begin{tabular}{cccc} 
& $\begin{array}{c}\text { Vertical Jump } \\
(\mathrm{cm})\end{array}$ & $\begin{array}{c}\text { Long Jump } \\
(\mathrm{cm})\end{array}$ & $\begin{array}{c}\text { Handgrip } \\
(\mathrm{N})\end{array}$ \\
\hline TD & $27.1 \pm 6.2$ & $163.2 \pm 28.7$ & $0.82 \pm 0.23$ \\
\hline LMP & $22.2 \pm 5.9$ & $157.8 \pm 34.3$ & $0.66 \pm 0.23$
\end{tabular}


(a) Knee isometric extension at $90^{\circ}$

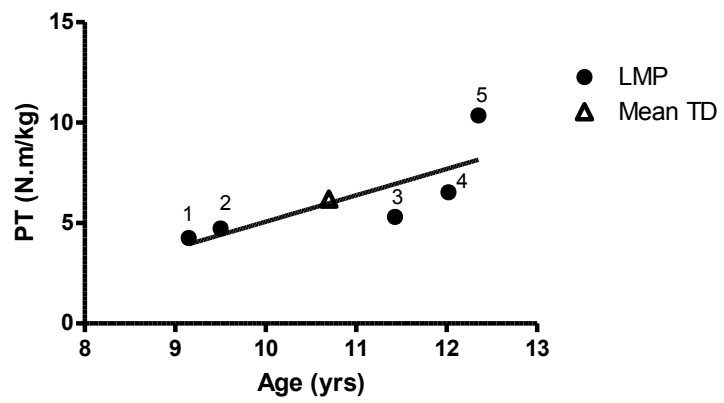

(b) Knee isokinetic extension at $60^{\circ} \cdot \mathrm{s}-1$

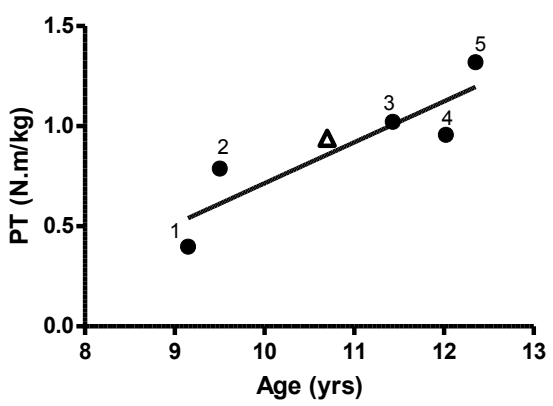

(d)

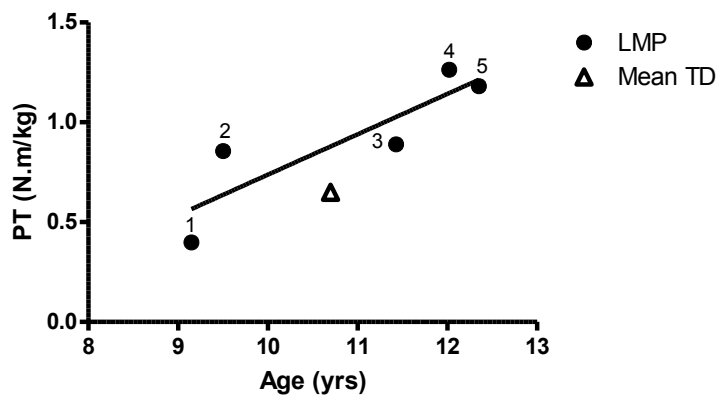

(c)
Knee isokinetic flexion at $60^{\circ} \cdot \mathrm{s}-1$

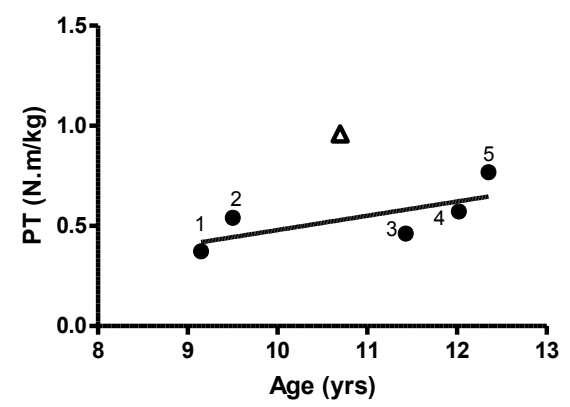

(e)

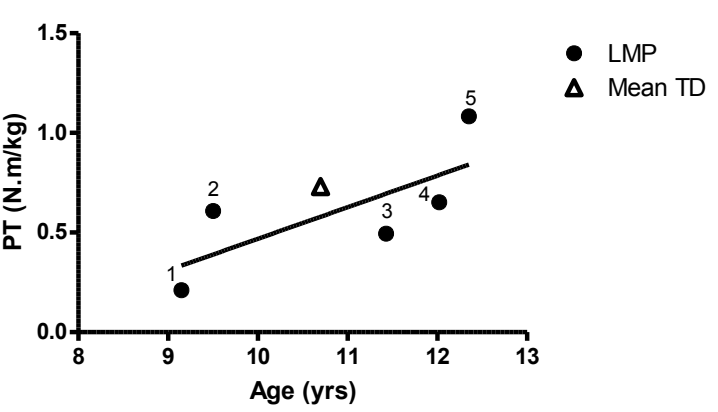

Figure 1: Individual children with low muscle performance (LMP) peak torque (PT) and typically developing children (TD) mean PT by age (yrs) in the following tasks: (a) knee isometric extension at $90^{\circ}$, (b) knee isokinetic extension at $60^{\circ} \cdot \mathrm{s}^{-1}$, (c) knee isokinetic flexion at $60^{\circ} \cdot \mathrm{s}^{-1}$, (d) knee isokinetic extension at $120^{\circ} \cdot \mathrm{s}^{-1}$, and (e) knee isokinetic flexion at $120^{\circ} \cdot \mathrm{s}^{-1}$. 
(a)

Handgrip

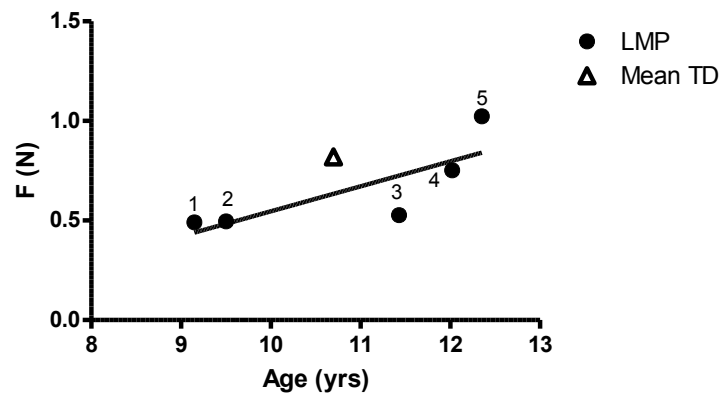

(b)

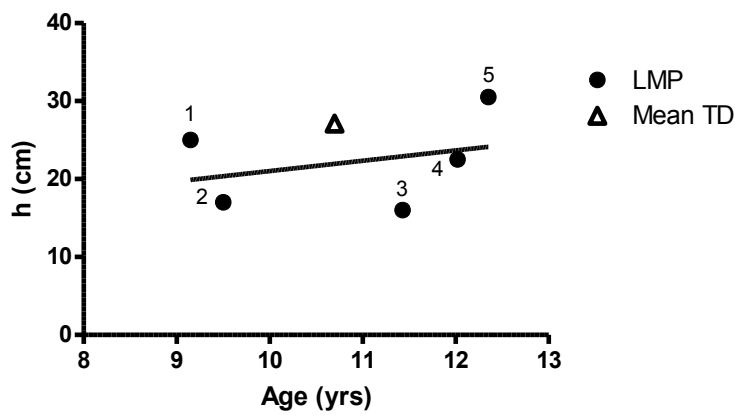

(c)

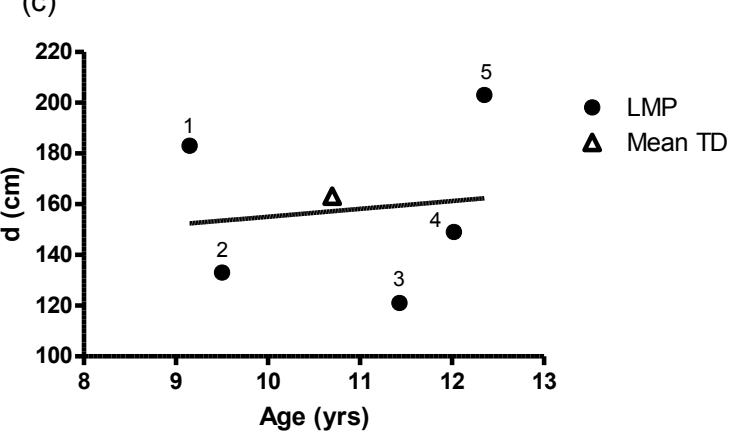

Figure 2: Individual children with low muscle performance (LMP) peak torque (PT) and typically developing children (TD) mean PT by age (yrs) in the following tasks: (a) Handgrip, (b) Vertical Jump, and (c) Long Jump.

\section{DISCUSSION}

The aim of this study was to explore descriptive mean scores in strength and power tests applied in children with low motor performance (LMP) as compared to their typically developing peers. We also individually analyzed the relationship of strength and power outputs with age progression. The group with LMP presented lower mean values than the TD group in all tests. Moreover, the tests scores showed that strength and power increased with age for those with LMP, with no apparent difference to the TD group, except for with knee flexion at $60^{\circ} \cdot \mathrm{s}^{-1}$. Due to the small number of individuals included on the group with LMP a comparison between groups in our study has no statistical significance. However, the findings demonstrate a trend of strength discrepancies between groups, and an improvement of the group with LMP strength with age at all tasks. These findings combined with previous studies reporting strength and power deficiencies in children with $D C D^{5,8,15-18}$ set the path for clarifying potential neuromechanical causes that may be causing motor coordination and control difficulties in children with LMP.

A plethora of studies have reported lower scores in fine and gross motor skills that involve physical fitness, balance and strength tests in children with this condition compared to same average age peers $5,6,8,14,18$. Although the information processing problems, programing and perceptual deficits they have may influence in the lack of coordination capability to accomplish tasks in the same level as normally developing children, neuromuscular aspects such as difference in level of muscular organization and muscle fiber distribution also seem to be relevant factors ${ }^{5}$. Raynor ${ }^{5}$ identified that children with $D C D$, for example, produced significantly lower levels of knee maximum extensor and flexor torque in isometric $\left(90^{\circ}\right)$ and isokinetic tasks at almost all speeds $\left(120^{\circ} \cdot \mathrm{s}^{-1}, 165^{\circ} \cdot \mathrm{s}^{-1}, 210^{\circ} \cdot \mathrm{s}^{-1}\right)$. These results were carried out together with increased levels of muscle coactivation, which may be associated with the planning, programing and muscle activation issues presented by these children ${ }^{5}$. Moreover, the analysis group-by-speed by age comparison demonstrated that the isokinetic extensor strength differed from 6 years old with DCD and TD peers at all speeds, but only at two speeds for 9 years old with DCD and TD children. This suggested that strength motor problems may decrease with age, and the lowest isokinetic speed is the easiest for their performance. Although we tested at the same isometric angle, but at one different $\left(60^{\circ} \cdot \mathrm{s}^{-1}\right)$ and one similar $\left(120^{\circ} \cdot \mathrm{s}^{-1}\right)$ isokinetic speed, our descriptive mean values are in accordance with Raynor's study 
when finding that the group with LMP produced lower levels of isometric and isokinetic knee strength than TD. Likewise, the children with LMP demonstrated greater extensor isokinetic strength at the lowest speed and our $x$-axis by age figures indicated a trend of strength increases of this group with age with no apparent difference compared to the TD group. These results can be related to a developmental delay, in which some children with LMP may "catch up" to their TD peers as their age increases ${ }^{1,5}$. However, Raynor's study did not show age by group interactions for the isokinetic flexor strength, relating this to an increase level of difficulty for this action phase due to ineffective movement organization and central programing delays they may have when performing knee flexion followed by extension contractions. Fong et al. ${ }^{16}$ also found that children with DCD have difficulties such as increased delayed time to achieve isokinetic knee flexor peak torque at $180^{\circ} \cdot \mathrm{s}^{-1}$. Our findings match with these results, by demonstrating that although children with LMP had strength increases with age their line of best fit was lower than mean TD scores by age at $60^{\circ} \cdot \mathrm{s}^{-1}$, which may also demonstrate a difficulty in performing flexion actions. This was not found for $120^{\circ} \cdot \mathrm{s}^{-1}$, and the different speed tested in our study may have influenced this dissimilarity between studies. Furthermore, subject 3 with LMP, who is around one year and a half older than the age mean for children with TD, had his lowest scores compared to the mean TD also at knee isokinetic flexion test at $60^{\circ} \cdot \mathrm{s}^{-1}$. Surprisingly, subject number 2 with LMP, who is around one year younger than the age mean for children with TD, presented greater scores than TD mean at isokinetic knee extension at $120^{\circ} \cdot \mathrm{s}^{-1}$. This may demonstrate that this task at this speed is less complicated for this subject to perform, and that strength production of children with LMP may be specific to the task, which may be more or less complex for them ${ }^{8}$. Nevertheless, our majority of strength and power findings are similar to these studies and this may be explained due to increased levels of co-activation, programing problems, lack of movement experience and difference in muscle-fiber distribution that children with LMP, such as with DCD condition, may have compared to average same age normally developed peers ${ }^{5,16}$.

Although gross motor strength problems appear to be clear, previous findings demonstrate that children with LMP tend to have predominantly less fine motor strength control and high variability when performing submaximal tasks $\mathrm{s}^{8,15}$. Oliveira et al. $^{8}$ reported that children with DCD did not differ from TD children in three different maximal isometric indexthumb tasks torque production, but had significantly larger torque variability in the constant submaximal task $(40 \%$ of maximal torque/force), which required the CNS to find solutions to a greater number of kinetic variables, involving a larger number of muscles to accomplish the task. This finding can be related to other studies that have also found higher variability and less steady force ${ }^{15}$, or delayed trajectory of finger torque control ${ }^{18}$, revealing fine motor strength difficulties in children with DCD. Pereira et al. ${ }^{17}$ also found that in children with DCD, grip hand force control varies and is impaired compared to controls. We did not directly test strength control in our study, however both groups were required to hold the hand dynamometer for 5 seconds during the handgrip test. The descriptive scores of both hands' strength summed demonstrated a lower average value for LMP compared to TD children. Although this does not measure variability of strength during a fine motor test, it may demonstrate that children with LMP did not produce the same level of strength and might have loosened or inconstantly grabbed the hand dynamometer during the entire requested time. This would be in accordance to the strength variability and weak control findings for children with DCD. However, to confirm this prediction handgrip strength variability would have to be measured in order to understand the impairments underlying the lack of handgrip strength of the group with LMP during this test. Similarly to the lower limbs isokinetic and isometric tests, the group with LMP presented a trend of handgrip strength increase and no apparent differences compared to the TD group mean scores by age. This demonstrates that they may also "grow out" their upper limb strength deficiencies.

The strength impairments presented by children with DCD may affect several difficulties they usually have in performing physical fitness tests $6,13,14,20$, such as explosive power performance tasks at the same level as normally developed peers ${ }^{13,21,22}$. This may be due to their difficulty in controlling and coordinating their body during functional motor tasks ${ }^{13}$. Ferguson et al. ${ }^{13}$ found no differences between children with DCD and TD children during isolated explosive movements (chest throwing and overarm throwing), but lower performance scores in whole body explosive movements tests, such as swing overarm throwing and standing long jump. In addition, both Kanioglou ${ }^{22}$ and $\mathrm{Hands}^{2}$ Larkin 9 studies reported poor performance on standing broad jump tests in children with DCD and in children with motor learning difficulties. Tsiotra et al. ${ }^{21}$ also found that children with DCD presented lower scores on vertical jump tests compared to TD peers. The poor whole body explosive performance observed during jump task performance may be attributed to the fact that these movements require a simultaneous coordination and timing of lower and upper limbs in order to generate power to jump ${ }^{13}$. We also found descriptive lower average results in the group with LMP as compared to TD children for both long jump and vertical jump tests. The long and vertical jumps require coordination, dynamic balance ${ }^{9,21,22}$ along with timing to release the energy stored during the preparation movement at the right time for a high scoring jump ${ }^{13}$. Children with DCD have been reported to present difficulties with all of these necessary physical elements for a good jump ${ }^{6,13}$, which may explain the low LMP average and high variability of results in our study. Subject number 3 with LMP also presented the lowest scores compared to mean TD at both jump tests, demonstrating that the delay of children with LMP in these complex tasks may persist during childhood compared to TD peers. However, similar to the strength tests, we identified a trend of increase in scores with age for the group with LMP. This may show their ability to improve their explosive power performance with age. Nevertheless, to the present date we are not aware of previous studies comparing the scores of individuals with LMP by age interaction in jump tests. 
The results demonstrated in our study complement several previous experimental studies and provide unique findings to validate the recommendation that strength and power exercises should be included in therapeutic programs for children with coordination and control difficulties 5,9,13,21,22. The European Academy for Childhood Disability (EACD) recommendation for clinical practice is an approved medical and therapeutic guideline of definition, diagnosis and intervention of children with $D C D^{12}$. This guideline states that strength exercises may be effective for children with $D C D$, but more studies are needed to determine the conditions and which kind of strength exercises should be performed. Moreover, no recommendations for neuromuscular coordination and power improvements were described in these guidelines. To our knowledge, only two studies have investigated the effectiveness of strength training in motor coordination improvements in children with LMP. Menz et al. ${ }^{11}$ reported that 24 strength training sessions of isolated pulley weight system simple joint movements of 3 sets of 30 repetitions with $0.5 \mathrm{~kg}$ increase per session improved complex motor skills, balance and coordination of a 6-year-old child suspected of having DCD. The training followed the Universal Exercise Unit (UEU), including most exercises in the supine and seated positions to improve the child's ability to more competently use feedforward control and not compensate during simple joint movements. Kaufman \& Schilling ${ }^{10}$ also found positive results for improvement of gross motor skills along with strength and proprioception improvements after a 12 week body and free weight strength training program for a 5 -year-old child with DCD; a case-study. The program was performed twice a week with one set of 6 repetitions and weight increases according to the child's level of fatigue and tolerance. They used a sequence based on the Kraemer \& Ratames ${ }^{23}$ publication, which suggests the use of primarily multi-joint movements, large muscle group exercise and rotating opposing antagonist and agonist exercises. Although Menz et al. ${ }^{17}$ used mainly isolated simple joint movements due to better stabilization for children with DCD in strength performance, Kaufman \& Schilling ${ }^{10}$ preferred a multi-joint exercise program of free and body weight resistance, which may approximate more to dynamic muscle function of everyday tasks and lead to increased neuromuscular motor learning in children ${ }^{10,23,24}$. This may also diminish their motor coordination problems ${ }^{10}$. Since motor unit recruitment and strength production in children is mainly associated with motor coordination and learning ${ }^{10,24}$, strength conditioning with a variety of isolated single and multi-joint exercises ${ }^{24}$ should be considered in therapeutic programs for children with LMP to improve strength and power along with their affected gross and fine motor skills in activities of daily living. Strength training programs with a variation of isolated pulley weight, medicine balls, free weights, body weight, cords, elastic bands, and plyometric jumps and hops set in creative play activities supervised by competent physical professionals ${ }^{24}$ may improve neuromuscular strength and power in these children. These exercises, if associated with overweight treatment and fine motor exercises (e.g. fingers and handgrip tasks), may improve their motor coordination and neuromuscular learning skills.

The limitations of this study include the small sample of children with LMP, gender and weight differences between and among groups (which could affect the normalized strength and power scores), and possible children growth/maturation differences regardless of age. However, all our results can be strongly related to significant previous studies in children with LMP and DCD. This leads to a significant discussion about the cause and effect issues related to difficulties in motor control and coordination and the necessity of strength and power exercises in guidelines and therapeutic programs for children with LMP.

\section{CONCLUSION}

In summary, our results suggest that children with LMP present lower average strength and power scores compared to TD peers, which may be affecting their ability to perform gross and fine motor skills, although they may "catch up" with age progression. The underlying mechanisms of these low scores are still unknown. However some authors have suggested that the reasons may be due to increased and low control of neuromuscular activation ${ }^{5}$, increased co-contraction of antagonist muscles groups ${ }^{11}$, weak proprioception aspects ${ }^{10}$, and deficits in several areas of the brain ${ }^{8}$. While the number of children included in the group with LMP was limited, we have found relevant and strongly related results similar to those found in the literature, which may suggest strength and power training programs for motor control, coordination and neuromuscular improvements.

\section{REFERENCES}

1. Zwicker J. G., Missiuna C., Harris S. R, \& Boyd L. A. (2012). Developmental coordination disorder: a review and update. European Journal of Pediatric Neurology, 16, 573-581.

2. Cairney J., \& Veldhuizen S. (2013). Is developmental coordination disorder a fundamental cause of inactivity and poor health-related fitness in children?. Developmental Medicine and Child Neurology, 55 (4), 55-58.

3. Piek J. P., \& Dyck M. J. (2004). Sensory-motor deficits in children with developmental coordination disorder, attention deficit hyperactivity disorder and autistic disorder. Human Movement Science, 23, 475-488. 
4. Hendrix C. G., Prins M. R., \& Dekkers H. (2014). Developmental coordination disorder and overweight and obesity in children: a systematic review. Obesity Reviews, 15, 408-423.

5. Raynor A. J. (2001). Strength, power, and coactivation in children with developmental coordination disorder. Developmental Medicine and Child Neurology, 43, 676-684.

6. Geuze R. H. (2005). Postural Control in Children With Developmental Coordination Disorder. Neural Plasticity, 12, 183-196.

7. Zhu Y. C., Cairney J., Li Y. C., Chen W. Y., Chen F. C., \& Wu S. K. (2014). High risk for obesity in children with a subtype of developmental coordination disorder. Research in Developmental Disabilities, 35, 1727-1733.

8. Oliveira M. A., Shim J. K., Loss J. F., de Souza Petersen R. D., \& Clark, J. E. (2006). Effect of kinetic redundancy on hand digit control in children with DCD. Neuroscience Letters, 410, 42-46.

9. Hands B. P., \& Larkin D. (2006). Physical fitness of children with motor learning difficulties. European Journal of Special Needs Education, 21, 447-456.

10. Kaufman L. B., \& Schilling, D. L. (2007). Implementation of a strength training program for a 5-year-old child with poor body awareness and developmental coordination disorder. Physical Therapy, 87, 455-467.

11. Menz S. M., Hatten K., \& Grant-Beuttler, M. (2013). Strength training for a child with suspected developmental coordination disorder. Pediatric Physical Therapy, 25, 214-223.

12. Blank R., Smits-Engelsman B., Polatajko H., \& Wilson P. (2012). European Academy for Childhood Disability (EACD): recommendations on the definition, diagnosis and intervention of developmental coordination disorder (long version). Developmental Medicine and Child Neurology, 54, 54-93.

13. Ferguson G. D., Aertssen W. F., Rameckers E. A., Jelsma J., \& Smits-Engelsman B. C. (2014). Physical fitness in children with Developmental Coordination Disorder: measurement matters. Research in Developmental Disabilities, 35, 1087-1097.

14. Volman M. J., Laroy M. E., \& Jongmans M. J. (2006). Rhythmic coordination of hand and foot in children with Developmental Coordination Disorder. Child: Care, Health and Development, 32, 693-702.

15. Smits-Engelsman B. C., Westenberg Y., \& Duysens J. (2008). Children with developmental coordination disorder are equally able to generate force but show more variability than typically developing children. Human Movement Science, 27, 296-309.

16. Fong S. S., Ng S. S., \& Yiu B. P. (2013). Slowed muscle force production and sensory organization deficits contribute to altered postural control strategies in children with developmental coordination disorder. Research in Developmental Disabilities, 34, 3040-3048.

17. Pereira H. S., Landgren M., Gillberg C., Forssberg H. (2001). Parametric control of fingertip forces during precision grip lifts in children with DCD (developmental coordination disorder) and DAMP (deficits in attention motor control and perception). Neuropsychologia, 39, 478-488.

18. King B. R., Clark J. E., \& Oliveira M. A. (2012). Developmental delay of finger torque control in children with developmental coordination disorder. Developmental Medicine and Child Neurology, 54, 932-937.

19. Holmes J. R., \& Alderink G. J. (1984). Isokinetic strength characteristics of the quadriceps femoris and hamstring muscles in high school students. Physical Therapy, 64, 914-918.

20. Batey C. A., Missiuna C. A., Timmons B. W., Hay J. A., Faught, B. E. \& Cairney, J. (2013). Self-efficacy toward physical activity and the physical activity behavior of children with and without Developmental Coordination Disorder. Human Movement Science.

21. Tsiotra G. D.; Nevill A. M., Lane A. M., \& Koutedakis, Y. (2009). Physical fitness and developmental coordination disorder in Greek children. Pediatric Exercise Science, 21, 186-195.

22. Kanioglou A. (2006). Estimation of physical abilities of children with developmental coordination disorder. Studies in Physical Culture and Tourism, 13, 25-32.

23. Kraemer W. J., \& Ratamess N. A. (2004). Fundamentals of resistance training: progression and exercise prescription. Medicine and Science in Sports and Exercise, 36, 674-688.

24. Faigenbaum A. D. (2000). Strength training for children and adolescents. Clinics in Sports Medicine, 19, 593-619.

Citation: Ruas CV, Punt C, Pinto RS, Oliveira MA. (2014) Strength and power in children with low motor performance scores: a descriptive analysis. BJMB 8 (1).

Editor: Joao Barros, California State University. Fullerton, CA. USA

Copyright: (C) 2014 Foden et al and BJMB. This is an open-access article distributed under the terms of the Creative Commons Attribution-NonCommercial-NoDerivatives 4.0 International License which permits unrestricted use, distribution, and reproduction in any medium, provided the original author and source are credited.

Funding: This study was not supported by any fellowship or funding.

Competing interests: The authors have declared that no competing interests exist.

Download: http://socibracom.com/bjmb/index.php/bjmb/issue/view/10 